\title{
Coronal linear threads: W-L radiation of supra-thermal streams
}

\author{
S. Koutchmy ${ }^{1}$ and A. G. Nikoghossian ${ }^{2,3}$ \\ 1 Institut d'Astrophysique de Paris, CNRS, 98bis bd. Arago, 75014 Paris, France \\ e-mail: koutchmy@iap.fr \\ 2 Byurakan Astrophysical Observatory, 378433 Byurakan, Armenia \\ e-mail: narthur@bao.sci.am \\ 3 Isaac Newton Institute of Chile, Armenian Branch
}

Received 9 July 2002 / Accepted 28 August 2002

\begin{abstract}
The paper aims at revealing the role of the Compton effect when considering the W-L scattering of the solar photospheric radiation by coronal supra-thermal streams. We first document several examples of well observed cases of linear W-L coronal threads extending above flaring active regions which are good candidates to give the signature known from the interpretation of radio type III bursts. The height-dependent model problem of Compton scattering on the beam of fast electrons gyrating around the lines of force of the magnetic field is considered. The resulting change in frequency averaged over the beam and the solid angle, within which the photospheric radiation falls, is computed for both sunward and antisunward directed streams. The dependence of the effect on the height above the solar surface, the speed of electrons and the slope angle is discussed. For illustration, we consider the case, in which the frequency distribution of the incident radiation is Planckian. The effective value of the cross-section of interaction is compared with that for the Thomson scattering. In particular, we conclude that, depending on the angle between the directions of the initial outburst and the magnetic field, the streams moving away from the Sun may produce drifts in frequency to the shortwave, as well as to the longwave domains of the spectrum. The effect may become essential even for moderate energies of fast electrons, and it should be measurable using precise photometric color index determination, if only the fractional density of fast electrons is not too small.
\end{abstract}

Key words. Sun: corona - activity - magnetic fields - particle emission

\section{Introduction}

For a long time high resolution W-L eclipse images taken near the time of the solar maximum of activity did show multiple thread-like streams and/or straight and fine "diverging" rays of unknown origin. This structure is not anchored at the surface of the Sun but appears as a straight linear structure starting from a fraction of the solar radius and was sometimes called rays or "fan-streamer" (Vsessviatski \& Bougoslavskaya 1944; Newkirk 1967; Koutchmy 1969, 1977). They are typically seen above limb active regions which produced flares or at least subflares before.

Coronal streams are one of the intriguing short-lived phenomena emerging from the magnetically dominated part of the inner atmosphere of the Sun; their typical linear structure, their large aspect ratio, and their dispersed directions with respect to the local radial direction strongly suggest that they largely ignore the outer background of the general coronal magnetic field which is usually computed using current-free or force-free extrapolations of the measured magnetic field at the solar surface. They probably represent fluxes of supra-thermal electrons (with velocities $v$ up to $10^{5} \mathrm{~km} \mathrm{~s}^{-1}$ ) moving partly along spiral trajectories. More complex MHD processes (reconnections) due to forces of magnetic, gravity and gas-dynamical origin are

Send offprint requests to: S. Koutchmy, e-mail: koutchmy@iap.fr also at work, where magnetic singularities (null-field locations) appear inside the corona.

Quite similar streams were considered by radioastronomers for a long time (see, e.g., Hartz 1969; Fainberg \& Stone 1971; Pick \& van Den Oord 1990; Thejappa et al. 1999) to explain plasma emissions which drift very rapidly in frequencies and produce type III bursts. The beams induce plasma oscillations at the local plasma resonance frequency which depends on the local density of electrons. A selffocusing mechanism is not excluded to keep the particles confined in the beam. Such mechanism was recently discussed by Lorrain \& Koutchmy (1996) on the context of spicule studies. It is usually assumed that the propagation of the beams occurred in the region of the intermediate corona, e.g., from 0.25 to several radii from the surface of the Sun but always above flaring regions (note that we do not claim that linear streams are produced by flares). The usually observed effect of type III bursts is the very fast longwaveward drift of the emission frequency which is believed to be connected with the generation of electron beams travelling antisunward, i.e., in the direction of decreasing density. However, some observational data reported during the past few years (see, e.g., Aschwanden et al. 1995; Klein et al. 1997; Melendez et al. 1999) point to the accelerated electrons over a short time scales and travelling also toward the solar surface, thus 
producing a frequency drift to the shorter wavelengths. This new radio diagnostic strongly suggests that the acceleration site of particles is definitely situated well above the surface.

During a decade the most popular dynamical phenomenon which has been considered as a possible source of "nonthermal" solar wind has been coronal holes and the dramatic CMEs (Coronal Mass Ejections). However, CMEs are only episodically related to active region flares; filament eruptions and escaping prominences are more typical phenomena of the CMEs. They are indeed believed to be not directly related to flares (Harrison 1986); sometimes it has even been suggested that a CME can induce a flare. There is no doubt that at least big flares produce large disturbances of the magnetic field of the surrounding corona as well as large flows of energetic particles in the Mev and up to the $\mathrm{GeV}$ range (neutrons and energetic muons are recorded with Cosmic Rays Monitors at the time of a flare, etc.). However, even the largest flares, like the 2000 Bastille day flare, produce little changes in the photospheric magnetic field (see Kosovichev \& Zharkova 2001). Large changes are occurring in the above lying corona, and the higher are the layers, the larger are the topological changes (see, e.g., the movies from the TRACE mission).

The ubiquitous optical fine rays seen above active flaring regions are also excellent candidates for considering a model of the interaction of the beam of relativistic electrons with the illuminating photospheric radiation when the $\mathrm{W}-\mathrm{L}$ corona is analysed.

The question we discuss in this paper is whether the Compton scattering effects (CSE) are important in interpreting the radiation of streams observed in optical and shorter wavelengths regions of the spectrum, and could be considered as a new potential diagnostics of non-thermal particles. To our knowledge, this point was never specifically considered by theorists, although the contribution of this radiation to the theory of the solar wind is certainly of great interest. Obvious implications will follow for stellar atmosphere physics when flares are important. The theoretical investigation of the coronal CSE assumes a study of the Compton interaction of the solar photospheric radiation with fast electron beams assuming curved trajectories of their motion. Due to the complex geometry of the photon-electron interaction, the problem requires a separate treatment, which is different from that when deriving the so-called redistribution function appearing in the transfer equation and considered by Rybicky (1979), Nikoghossian \& Haruthyunian (1980), Haruthyunian \& Nikoghossian (1981), Aharonian \& Atoyan (1981). The paper deals with the Compton scattering of photospheric photons on the beams of electrons gyrating in the magnetic field. The outline of the paper is as follows. We start, in Sect. 2, by presenting several examples of well-observed cases of linear W-L coronal supra-thermal streams. In Sect. 3, we derive an analytical expression for differential cross-section describing the photonelectron interaction in the observer's frame (in which the electron is moving). The description of the geometrical model and derivation of an explicit formula for the mean frequency change resulting from the single event of the Compton scattering are given in Sect. 4. The results of numerical calculations presented in Sect. 5 reveal the dependence of the mean frequency change

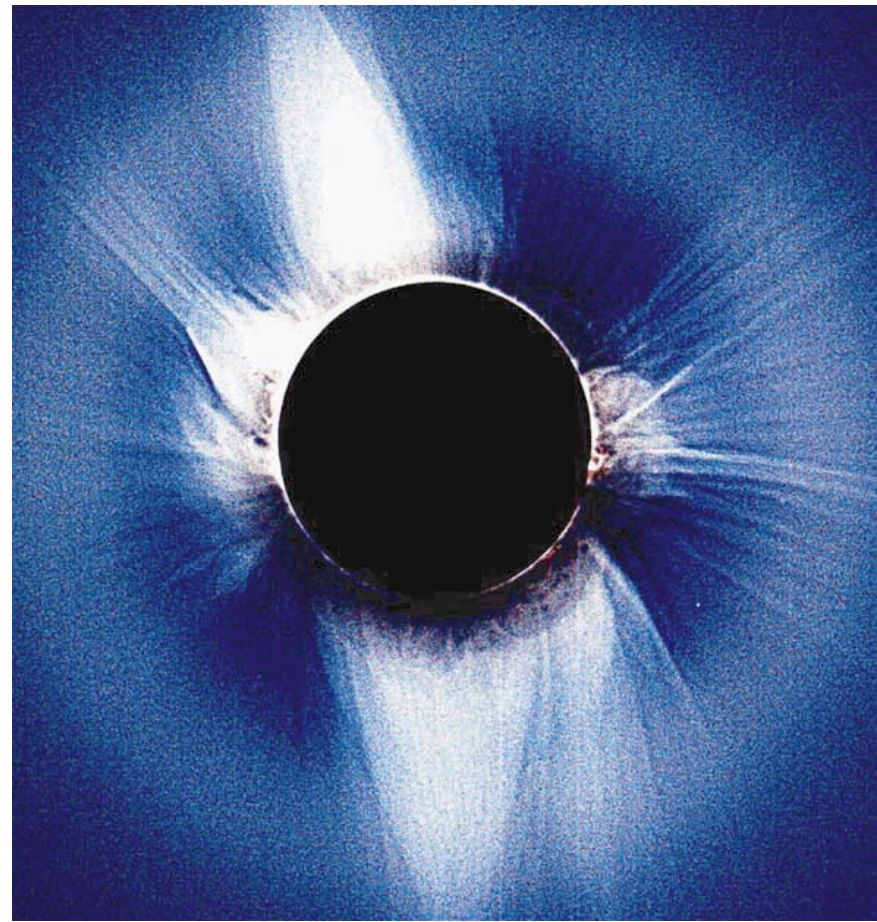

Fig. 1. The active corona as seen in W-L through a radial neutral filter suppressing the large radial gradient of intensities. The region above the West limb (at right) is overlying a largely flaring active regions. Many diverging linear or quasi-linear rays or threads can be identified (from a color image by the Institut d'Astrophysique de Paris team-CNRS, taken from the basement of the CFHT dome on the Mauna-Kea on July 11, 1991).

on the height, the slope angle of the beam and the energy of electrons. To estimate the effect of scattering, the value of the averaged cross-section for fast electrons is compared to that for electrons at rest (Thomson scattering limit). The observational aspect of the obtained results is discussed.

\section{Observational evidence of coronal W-L linear threads}

The fine structure of the corona was noticed since the very early eclipse observations made both visually and photographically. Due to high radial gradient of the coronal radiation it is not easy to distinguish thin radial details on photographs. However, in an early extended paper, Eddy (1973) analysed a very well recorded long linear structure of typically $10^{\prime \prime}$ width seen in the 1922 corona photographed on large eclipse plates. A good reproduction of one of the plates can be found in the popular book "A New Sun" under the Eddy (1979) direction, p. 84. This linear thread called by Eddy a coronal ray, extended from $h \approx 1.2$ to $h \approx 3$ solar radii and its transverse width kept almost constant. Its lifetime was more than $30 \mathrm{~min}$. From our experience (Koutchmy 1969, 1977), such ray is typical for the fine structure of the corona above a rather developed active region with an enhancement at the base, which means a great probability of flaring activity before, and after. 


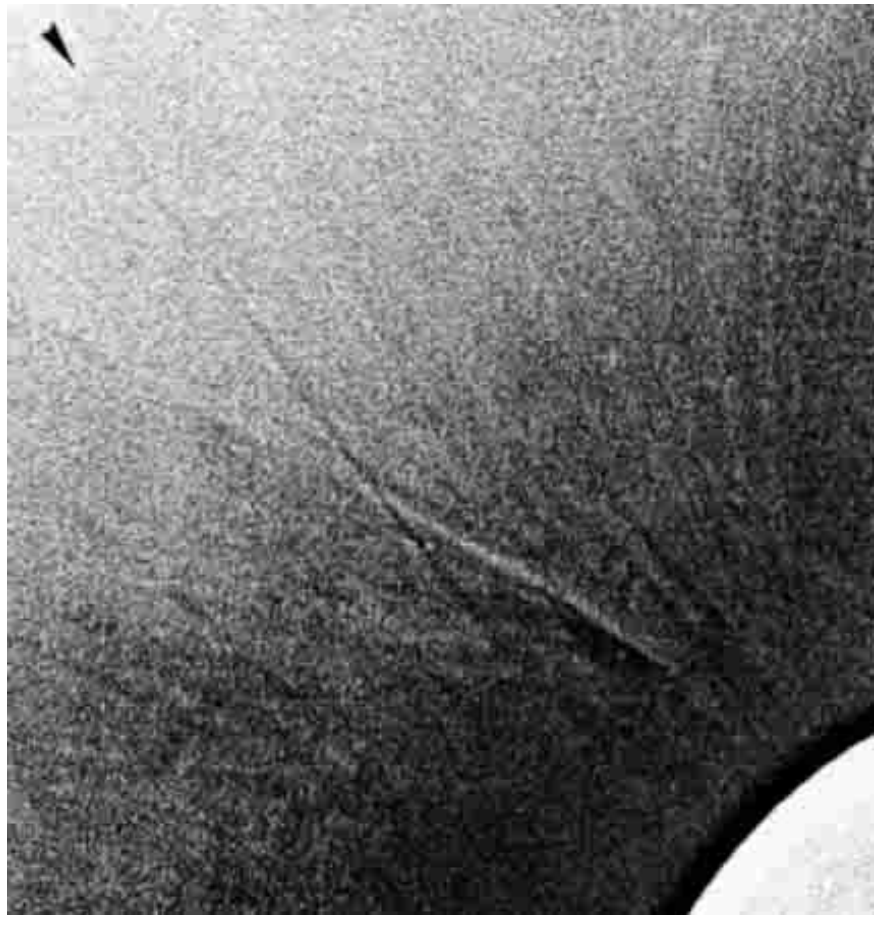

Fig. 2. Early observation of a coronal linear (in dark) ray-thread showing a large aspect ratio. This is a greatly magnified negative print made from a duplicate of an original $30 \times 30 \mathrm{~cm}$ glass eclipse plate obtained by the team of the Kiev University (Ukraine) using a $10 \mathrm{~cm}$ diameter lens of 10 meters focal length and $30 \mathrm{~s}$ exposure time. We processed a little bit the duplicate to partly remove the radial gradient of intensities, and reduce modulations of low spatial frequencies. The part which is shown here is situated above the E limb of the March 7, 1970 active corona.

Now we present some selected material pointing out to those properties. Figure 1 is a print of one of the radially filtered image we got in 1991 near the time of the maximum activity. It is showing a largely "open" corona above the W-limb (to the right) where an extended in longitude active region is seen in projection above the limb. From that region we recorded many fine rays extended like a fan and barely resolved. It is clear that this region, known as the most productive in flares during the cycle 22 one month before the eclipse, is overlaid by a large number of divergently extended linear rays or threads. It is not possible to distinguish definitely one of them because of the large overlapping effect produced when structures are projected over the plane of the sky. Let us notice that a rather compact condensation can be seen near the apparent point of convergence of these rays and that the condensation is reminiscent of the post-flare loops which are getting weaker and are not resolved in this image.

Diverging rays above a flaring region were described by Koutchmy as early as in 1969 and some photometric data were given from his W-L pictures made with a radial filter. However, the best studied ray or linear thread is probably a well recorded feature in the background of the active corona of March 7, 1970 eclipse, above the E-limb. Figure 2 shows some processed print of the region, as obtained from the best duplicate we did using the Kiev University plates made by N. Dzubenko. Other

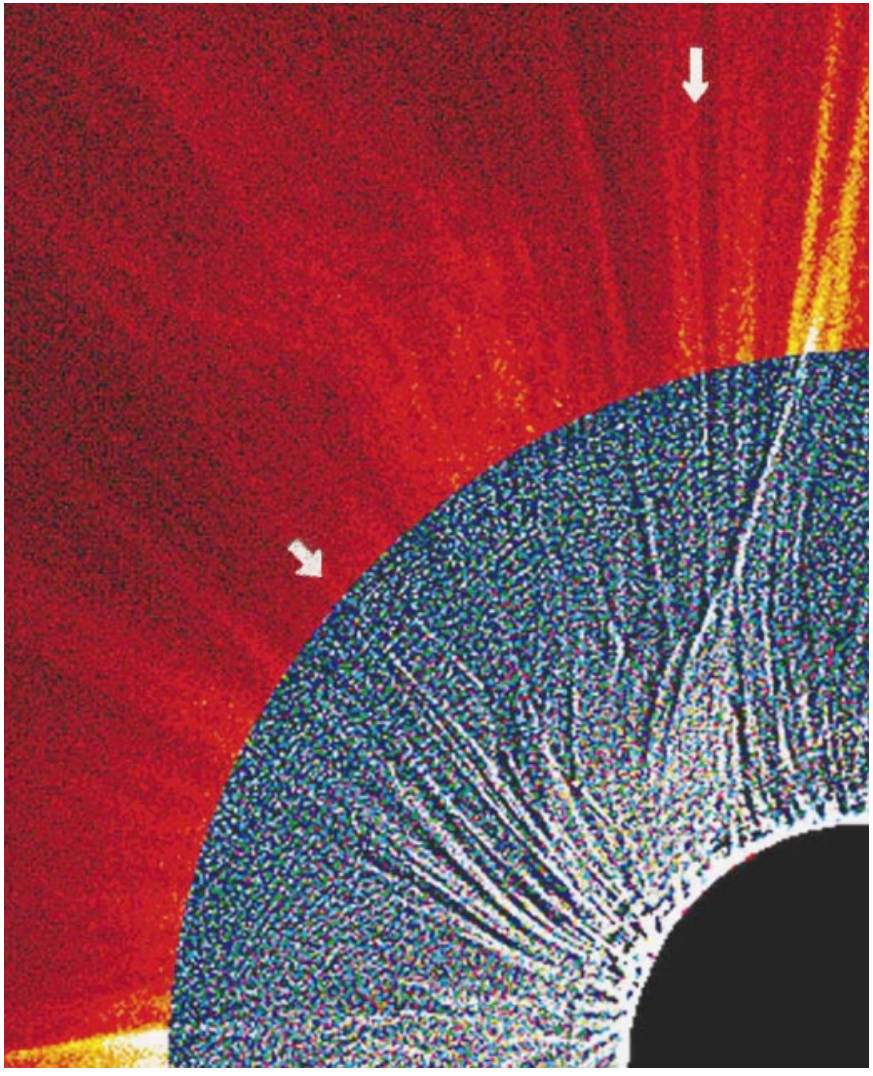

Fig. 3. Selected part of a composite (inner part is a W-L compressed image taken from Iran by the team of the Institut d'Astrophysique de Paris-CNRS using a radial filter; outer parts is the Lasco C2 image from SOHO taken simultaneously from space on July 11, 1999) to show the NE limb, right above a coming flaring active regions. High resolution images of the more inner parts of this limb did show many loops. Arrows are indicating the most obvious linear rays-threads. The one raising above the $\mathrm{N}$-pole region was seen on both groundbased and spaceborn images.

calibrated pictures were also made at lower resolution by one of the authors and discussed in Koutchmy (1987).

As was mentioned, the linear thread or ray extends over more than a solar radius and keeps its width approximately constant. Dzubenko et al. (1970) performed some photometric evaluation of the $F W H M$ and we repeated the work; typical values of about $15^{\prime \prime} \pm 5^{\prime \prime}$ were deduced. The apparent cross section is not constant along the ray but it is difficult to claim that this is just due to a variation of the true cross-section of the ray. Another possible interpretation is to assume variable electron density filling the structure or even in term of twist of a non circular section. What is the most conspicuous is the linearity of the thread: it is really difficult to detect any departure from linearity in the part which is well recorded as an individual ray. It is telling us that the formation of the ray is probably such that the surrounding background magnetic field does not influence the process, which is rather surprising unless the beam of particles which produced this structure is energetic enough to have a great "rigidity", a situation quite similar to that we meet when considering the rigidity of cosmic rays which are not deflected by the Earth magnetic field. Such beam of 
particles could also produce a self-focussing along the trajectory. The last example we selected is from one of the latest W-L image made with a radial filter at the Aug. 11, 1999 eclipse in Iran. The processed image of the corona observed again at the time of maximum of activity was precisely correlated with a cleaned and processed Lasco C2 image obtained almost simultaneously. Several example of linear threads can be identified over the field, some of them on both the groundbased and the spaceborne images, especially above limb active regions. Figure 3 is a part of the composite to show at best the linear threads and to point out 2 good examples of rays. This last example seems to confirm that the possibility exists to observe at least some parts of the linear rays and threads on Lasco C2 images which is a potentially very interesting circumstance.

Taking into account the morphological parameters ( $F W H M$; length) of the rays (threads) shown in Figs. 2 and 3, we deduce a typical aspect ratio of order of 100 , without removing the effect of the instrumental and atmospheric smearing present during the imaging process which can only reduce the value of the aspect ratio. Such high value of the aspect ratio tells us about: i). the effectiveness of the confinement which should be magnetic; ii). the low probability to explain this structure as due to a rather special geometrical projection effect, as suggested by the earlier observers, (see Billings 1966), which would make an apparent very narrow edge when a sheet (or 2D surface) is seen exactly edge-on. Accordingly, we favor a model of ray implying a beam of collectively accelerated particles with, eventually, a process of self-focussing or channeling, along a part of their travel through the corona (see other examples given by Lorrain \& Koutchmy 1996). The occurrence of many rays above an active region which produces several flares, see Fig. 1, would then find a natural explanation: rays could be the result of reconnection processes occurring before and after the flare in the low corona. Similar processes have been proposed to explain SXR jets, see, e.g., Yokoyama \& Shibata (1996). As was above said, the beams of relativistic electrons were indeed assumed by radio-astronomers for a long time to explain type III radio bursts. Recently, beams of collimated energetic electrons of flare origin were described up to the interplanetary medium, see Buttighoffer (1996) such that we find that optical rays we consider here have probably many counterparts. The range of velocities of electrons inside linear rays-threads can be very roughly evaluated from the aspect ratio we got before. The typical speed of thermal electron in a 2 millions degrees corona is $6000 \mathrm{~km} \mathrm{~s}^{-1}$ or $c / 50$. Assuming the magnification in speed is like the distribution of directions of motions, we see that the real speed should be magnified by a factor of order of $100^{2} / 2$ which is probably non-sense because the aspect ratio is not a pitch angle; a collimation/focussing or channeling is at work. We get indications that the supra-thermal particles could be relativistic. Let us remind that radio-astronomers measure a value of order of $c / 3$ to explain type III bursts. We guess that, in case of optical rays with 30 min or more lifetime (as evidenced from the comparison of images taken at eclipses and from Lasco C2 images), a reasonable range of speeds is probably between $c / 3$ and $c / 20$. This paper will not propose any model of rays; our ambition is just to look at the color effects produced when fast electrons are considered and find out a solid diagnostic to evaluate their speed inside those linear rays-threads.

\section{Compton scattering}

Compton scattering provides a means by which electrons interact with photons without changing the photons number. A differential cross section valid for any system reads (Berestetski et al. 1989)

$\mathrm{d} \sigma=8 \pi r_{\mathrm{e}}^{2} \frac{\widetilde{m}^{2} U_{0} \mathrm{~d} t}{\left(s-\widetilde{m}^{2}\right)^{2}}$,

where

$$
\begin{aligned}
U_{0}= & \left(\frac{\widetilde{m}^{2}}{s-\widetilde{m}^{2}}+\frac{\widetilde{m}^{2}}{u-\widetilde{m}^{2}}\right)^{2} \\
& +\left(\frac{\widetilde{m}^{2}}{s-\widetilde{m}^{2}}+\frac{\widetilde{m}^{2}}{u-\widetilde{m}^{2}}\right)-\frac{1}{4}\left(\frac{s-\widetilde{m}^{2}}{u-\widetilde{m}^{2}}+\frac{u-\widetilde{m}^{2}}{s-\widetilde{m}^{2}}\right)
\end{aligned}
$$

$r_{\mathrm{e}}$ is the classical radius of electron, $\widetilde{m}=m c, m$ is the electron mass, $c$ is the velocity of light, and $s, u, t$ are the kinematic invariants defined as follows

$s=\widetilde{m}^{2}+2 p k, \quad u=\widetilde{m}^{2}-2 p k^{\prime}, \quad t=-2 k k^{\prime}$.

In writing Eq. (1) both the photon and electron are assumed unpolarized. Hereafter we adopt the commonly used notations: $k$ and $p$ are 4-momenta of photon and electron before interaction, while $k^{\prime}$ and $p^{\prime}$ are 4-momenta of photon and electron resulting from interaction. In terms of kinematic invariants Compton's formula has the form

$s+u+t=2 \widetilde{m}^{2}$

To proceed we need the explicit expressions for the scalar products appearing in Eq. (3)

$p k=h m v \eta, \quad p k^{\prime}=h m v^{\prime} \eta^{\prime}$,

$k k^{\prime}=\frac{h^{2}}{c^{2}} v v^{\prime}(1-\cos \theta)$,

where $h$ is Planck's constant, $v$ and $v^{\prime}$ are the initial and final photons frequencies, $\theta$ is the scattering angle; besides we have introduced

$\eta=\gamma\left(1-\beta \cos \psi_{i}\right), \quad \eta^{\prime}=\gamma\left(1-\beta \cos \psi_{f}\right)$,

where $\beta=|\boldsymbol{p}| / c, \gamma=\left(1-\beta^{2}\right)^{-1 / 2}$ is the relativistic factor, $\psi_{i}$ and $\psi_{f}$ are the angles composed by directions of incident and scattered photons with direction of the electron momentum. In view of Eqs. (3) and (5) one may rewrite Eqs. (1) and (4) in the form

$\mathrm{d} \sigma=\pi r_{\mathrm{e}}^{2} \frac{U \mathrm{~d} t}{2 x^{2} \widetilde{m}^{2} \eta^{2}}$,

where

$U=\left(\frac{1}{x \eta}-\frac{1}{x^{\prime} \eta^{\prime}}\right)^{2}+2\left(\frac{1}{x \eta}-\frac{1}{x^{\prime} \eta^{\prime}}\right)+\frac{x \eta}{x^{\prime} \eta^{\prime}}+\frac{x^{\prime} \eta^{\prime}}{x \eta}$ 


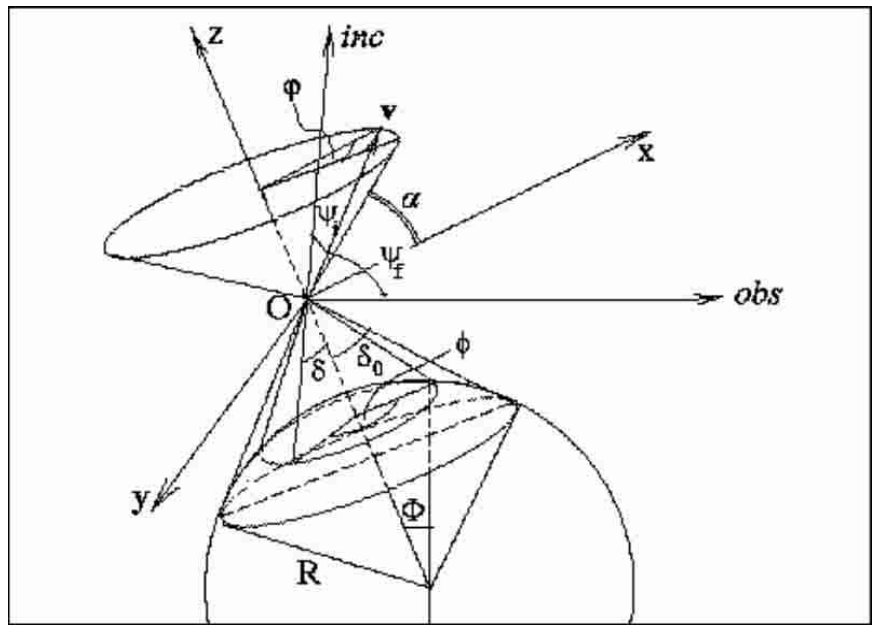

Fig. 4. The geometry of the Compton scattering of photospheric radiation on a single beam of fast electrons precessing around the radially directed magnetic field (axis $\mathrm{Oz}$ ). Outward motion corresponds to $\alpha>0$.

and

$x^{\prime}=\frac{x \eta}{\eta^{\prime}+x(1-\cos \theta)}$,

where $x=h v / m c^{2}, x^{\prime}=h v^{\prime} / m c^{2}$.

The relativistic invariant $t$ may be expressed through the invariant $x^{\prime 2} \mathrm{~d} \Omega=x^{\prime 2} \sin \theta \mathrm{d} \theta \mathrm{d} \varphi$ (see, Akhiezer \& Berestetski 1959) as follows

$\mathrm{d} t=m^{2} c^{2} x^{\prime 2} \mathrm{~d} \Omega$.

In view of Eqs. (7) and (9), Eq. (7) takes the form

$\mathrm{d} \sigma=r_{\mathrm{e}}^{2}\left(\frac{x^{\prime}}{x}\right)^{2} \frac{U}{2 \eta^{2}} \mathrm{~d} \Omega$,

where

$U=\frac{x \eta}{x^{\prime} \eta^{\prime}}+\frac{x^{\prime} \eta^{\prime}}{x \eta}-2 \frac{1-\cos \theta}{\eta \eta^{\prime}}+\frac{(1-\cos \theta)^{2}}{\eta^{2} \eta^{\prime 2}}$.

As far as we are interested in moderate values of electron velocities $(\beta \leq 0.4)$, the smallness of $x$ allows to simplify Eq. (11) to write

$\mathrm{d} \sigma=r_{\mathrm{e}}^{2} \frac{U}{2 \eta^{\prime 2}} \mathrm{~d} \Omega$

where now

$U=1+\left(1-\frac{1-\cos \theta}{\eta \eta^{\prime}}\right)^{2}$.

\section{The geometrical model}

Figure 4 schematically shows the geometry of the scattering process of a single beam of electrons gyrating around the radially directed lines of force of the magnetic field (axis $\mathrm{Oz}$ in Fig. 4). A rectangular Cartesian coordinate system is introduced in such a way that the line of sight lies in the plane $x O z$. Magnetic field makes a certain angle $\Phi$ with the perpendicular to the line of sight direction. This angle referred hereafter to as "the slope angle" takes positive values for inclinations towards the observer, and negative ones for the opposite direction. If we suppose that all the electrons involved in the beam are moving along similar trajectories (i.e. with the same thread of the spiral), the velocity vectors $v$ will then have the same projection on the axis $O z$. Hence, at a given height, the velocity vectors of the electron flux make one and the same angle $\alpha$ with the plane $x O z$, so that $\alpha$ specifies the pitch angle. The angles $\psi_{\mathrm{i}}$ and $\psi_{\mathrm{f}}$ determine the directions of incident (abbreviatedly marked in Fig. 4 by inc) and scattered photons. Further, the line of sight with impact parameter $h$ (referenced from the solar surface) intersects the electron beam at a point $O$ chosen as the origin of the coordinate system. From this point the solar disc is seen within a solid angle

$\Omega=2 \pi \int_{0}^{\delta_{0}} \sin \delta \mathrm{d} \delta$

where $\delta_{0}=\arcsin \frac{\cos \Phi}{1+(h / R)}$, and $R$ is the radius of the Sun.

The components of the unit vectors specifying the directions of the electron motion, as well as the directions of incident and scattered radiation, are respectively

$\boldsymbol{v}_{0}(\cos \alpha \cos \varphi, \cos \alpha \sin \varphi, \sin \alpha)$,

$\boldsymbol{n}(\sin \delta \cos \phi, \sin \delta \sin \phi, \cos \delta)$

$\boldsymbol{n}^{\prime}(\cos \Phi, 0, \sin \Phi)$

so that

$\cos \theta=\left(\boldsymbol{n}, \boldsymbol{n}^{\prime}\right)=\sin \delta \cos \phi \cos \Phi+\cos \delta \sin \Phi$,

$\cos \psi_{\mathrm{i}}=\left(\boldsymbol{n}, \boldsymbol{v}_{0}\right)=\sin \delta \cos \alpha \cos (\varphi-\phi)+\cos \delta \sin \alpha$,

$\cos \psi_{\mathrm{f}}=\left(\boldsymbol{n}^{\prime}, \boldsymbol{v}_{0}\right)=\cos \alpha \cos \Phi \cos \varphi+\sin \alpha \sin \Phi$.

In the particular case of $\delta_{0}=0$, Eqs. (3) lead to the results given by Nikoghossian \& Koutchmy (2001).

The quantity of interest for us is the mean value of the frequency change resulting from the interaction of a photon with the electron beam

$\frac{\left\langle x^{\prime}\right\rangle}{x}=\frac{\int x^{\prime} \mathrm{d} \sigma}{x \int \mathrm{d} \sigma}$.

The integration in Eq. (18) extends over the entire domain of variation of $\delta$ and the azimutal angles $\varphi$ and $\phi$. For a fixed $\delta$, the first of Eqs. (17) yields $\mathrm{d} \cos \theta=\sin \delta \cos \Phi \cdot \mathrm{d} \cos \phi$. With this in mind, we substitute Eq. (13) into Eq. (18) to obtain

$$
\frac{\left\langle x^{\prime}\right\rangle}{x}=\frac{\int_{0}^{\delta_{0}} \sin \delta \mathrm{d} \delta \int_{-1}^{1} \mathrm{~d} \zeta \int_{-1}^{1} U \frac{\eta \mathrm{d} \xi}{\eta^{\prime 3} \sqrt{1-\xi^{2}}}}{\int_{0}^{\delta_{0}} \sin \delta \mathrm{d} \delta \int_{-1}^{1} \mathrm{~d} \zeta \int_{-1}^{1} U \frac{\mathrm{d} \xi}{\eta^{\prime 2} \sqrt{1-\xi^{2}}}}
$$

where $\xi=\cos \varphi, \zeta=\cos \phi$. In writing Eq. (7), we neglect the limb darkening effect. 


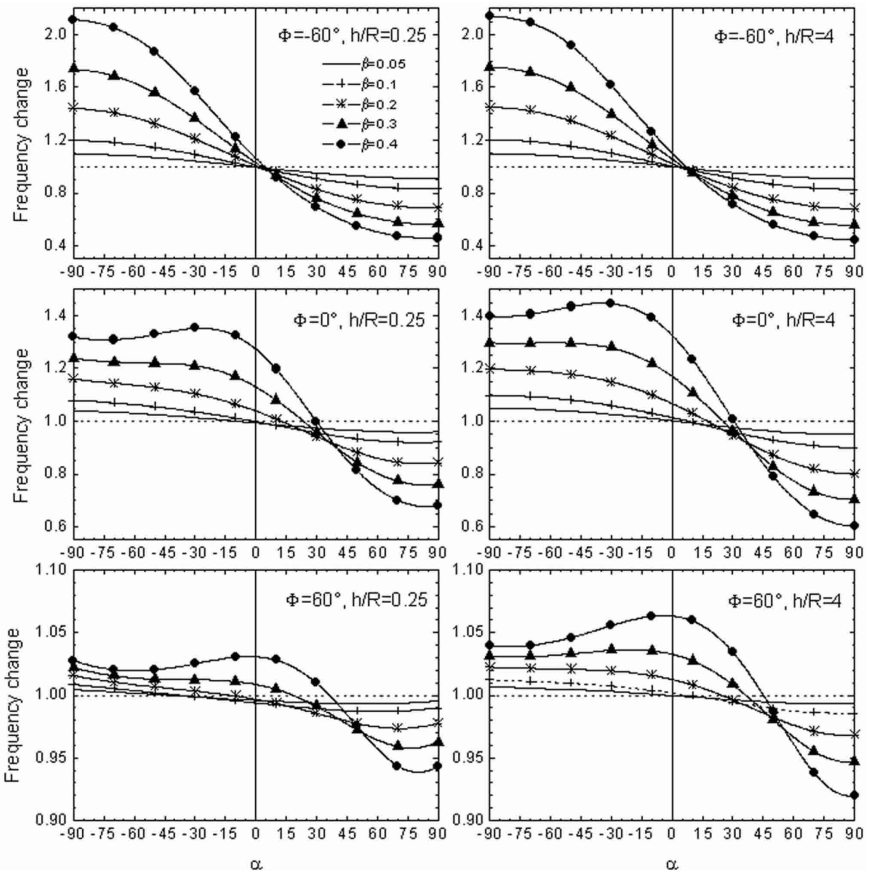

Fig. 5. Dependence of the frequency change on $\alpha$ for fixed values of $\beta$ and for different inclination angle $\Phi$. The dotted line corresponds to $\left\langle x^{\prime}\right\rangle / x=1$.

\section{Numerical results}

Figure 5 presents the graphs of the relative mean frequency change which result from the scattering on a single beam of moving electrons. Calculations made for two different heights $(h / R=0.25 ; 4)$ illustrate the height-dependence of the effect. The observing pattern for both heights is qualitatively in agreement with that described previously in the above-cited paper of the present authors, in which the incident radiation was supposed to be monodirectional. This is especially the case, as might be expected, at high distances from the Sun, for which the role of the solid angle becomes negligible (the case $h / R=4$ in Fig. 5). Here again drifts to the shortwave domain of the spectrum are mainly due to sunward directed beams. However, it is seen that such drifts may also be produced by electrons moving away from the Sun. This occurs for $\Phi \geq-\pi / 6$ and relatively small values of $\alpha$. It should be emphasized that the largest changes in frequencies are observed for negative slope angles, $\Phi$, i.e., for directions opposed to the observer. Note also that, in this latter case, the antisunward flows cause drifts to the longwave domain of the spectrum. Another feature to be noted is the saturation behavior of the frequency change at high distances. This level is attained differently depending on the slope angle $\Phi$ : for negative $\Phi$, the limiting values are established much faster, than in the opposite case. It is seen that, for $\Phi=-60^{\circ}$, only minor differences are observed between the results corresponding to the two values of the impact parameter, while the difference in heights over the solar surface is significant: $1.5 R$ $(h / R=0.25)$ and $9 R(h / R=4)$.

Figure 6 demonstrates the effect of Compton scattering on a single beam of electrons perpendicular to the line of sight $(\Phi=0)$ in case when the intensity of the incident radiation is

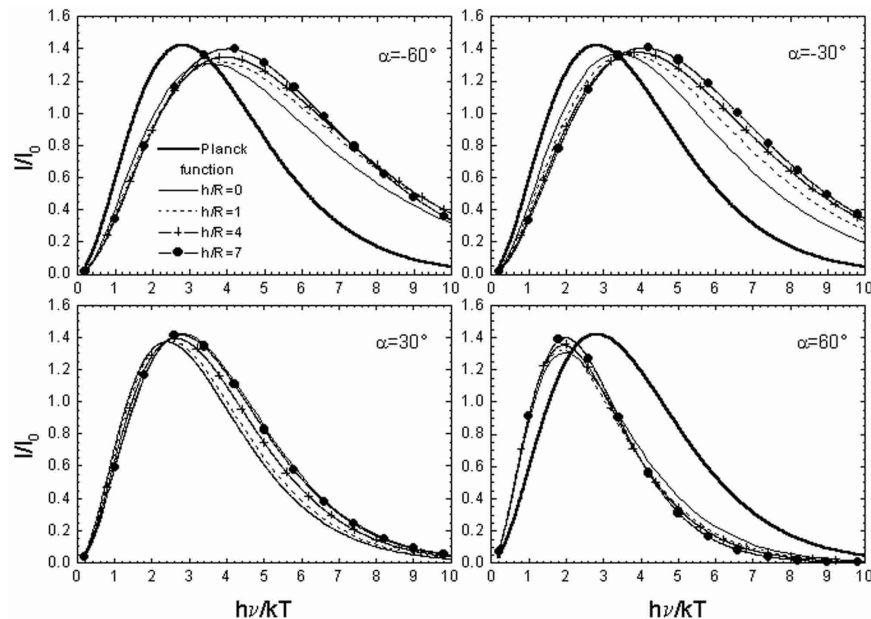

Fig. 6. The Compton scattering effect for $\Phi=0, \beta=0.4$ and for a set of indicated values of $\alpha$ and the impact parameter if the incident radiation is Planckian.

assumed to be given by the Planck function. The dimensionless intensity evaluated for the scattered radiation is

$I(x)=I_{0} x^{3} /\left[\exp \left(\frac{x}{r}\right)-1\right]$

where $x=h v / k T, I_{0}=2(k T)^{3} /(c h)^{2}, k$ is Boltsmann's constant, $T$ is the effective temperature, and $h$ is Planck's constant. The ratio $r=\left\langle x^{\prime}\right\rangle / x$ is found from Eq. (7). Calculations concern different heights for $\beta=0.4$ and the indicated values of $\alpha$. As before, for $\delta_{0}=0$, the maximum increase in the photon energy resulting from the scattering on outwards directed flows of electrons is observed for positive angles $\Phi$ and relatively small values of $\alpha(\alpha \leq \pi / 6)$, the latter being dependent on the energy of moving electrons. One can see that the largest drifts for positive $\alpha$ occur at low heights near the base of a stream, while for negative $\alpha$ the frequency drifts are greater at high distances. For both downward and outward directions of the streams' propagation, the displacement of the intensity maximum relative to the Planck function increases with an increase of the absolute value of $\alpha$. As might be expected, the higher the velocities of scattering particles, the greater the observed drift. For $\alpha=-\pi / 3$, the frequency of the intensity maximum at all heights shows an increase of about 1.07 times, if $\beta=0.1$, and $1.29(h=0)$ or $1.43(h / R=7)$ times, if $\beta=0.4$. These drifts particularly enhance the intensity observed in the optical domain of the spectrum. For instance, at $\lambda=5000 A$, the intensity grows by a factor 1.2 , if $\beta=0.1$, and, correspondingly, by a factor $1.56 \div 1.80$ (depending on the height), if $\beta=0.4$. Even greater are the longwards drifts, which occur when $\alpha$ is positive. For $\alpha=\pi / 3$, the frequency of the intensity maximum decreases by a factor of $1.07(\beta=0.1)$ and $1.4 \div 1.6(\beta=0.4)$. This reduces the intensity observed at $\lambda=5000 A$ by a factor of $1.28(\beta=0.1)$ and $2.6 \div 4(\beta=0.4)$. The effect of an ensemble of radially directed and overlapping streams is only slightly different from that described above providing for the dispersion is not in excess. This may become observable if only the fractional density of fast electrons and the proper cross-section are not too small. This point is considered in the next section. 

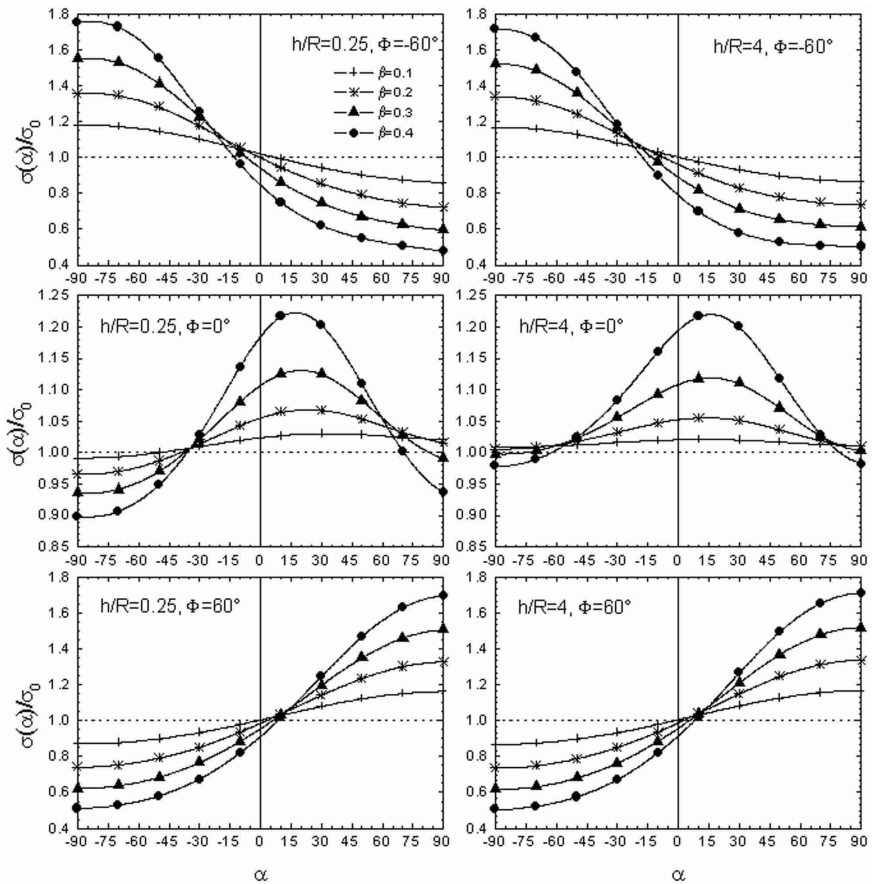

Fig. 7. The ratio of the averaged cross-section for Compton scattering to that of Thomson scattering for two heights and for the indicated values of $\Phi$.

\section{Cross-section and concluding remarks}

To give an idea of the observational aspect of the scattering effect, we consider the ratio of the averaged (over the solid angle $\Omega$ ) value of the cross-section evaluated for relativistic electrons over the value for electrons at rest (Thomson scattering limit). Both of them depend obviously on $\Phi$, at the same time the former is determined by two additional parameters $\alpha$ and $\beta$. Using Eq. (11) we have

$$
\frac{\sigma(\alpha, \Phi, \beta)}{\sigma_{\mathrm{T}}(\Phi)}=\frac{\int_{0}^{\delta_{0}} \sin \delta \mathrm{d} \delta \int_{-1}^{1} \mathrm{~d} \zeta \int_{-1}^{1} U \frac{\mathrm{d} \xi}{\eta^{\prime 2} \sqrt{1-\xi^{2}}}}{\pi \int_{0}^{\delta_{0}} \sin \delta \mathrm{d} \delta \int_{-1}^{1}\left(1+\cos ^{2} \theta\right) \mathrm{d} \zeta} .
$$

Integrations in the denominator are performed analytically to yield an explicit expression for $\sigma_{\mathrm{T}}$

$$
\begin{aligned}
\sigma_{\mathrm{T}}= & 2 \pi\left(1+\frac{1}{3} \cos ^{2} \Phi\right)\left(1-\cos \delta_{0}\right) \\
& +2 \pi \frac{1}{3}\left(1-\frac{4}{3} \cos ^{2} \Phi\right)\left(1-\cos ^{3} \delta_{0}\right) .
\end{aligned}
$$

The results of calculations obtained from Eq. (15) for several slope angles $\Phi$ and two values of impact parameters $(h / R=0.25 ; 4)$ are given in Fig. 7 . We see that the dependence on height is insignificant: only minor differences are exhibited in case of $\Phi=0$. We further conclude that the cross-section of interest to us may exceed that for Thomson scattering up to 1.8 (for $\beta=0.4$ ) times, as it is the case for $\Phi<0$ ) and sunward directed beams, as well as for $(\Phi>0$ and antisunward directed ones. The first of these processes shorten the wavelengths, while the second one, depending on $\alpha$, may produce drifts to both shortwave and longwave domains of the spectrum. This latter case is of primary importance from the point of view of supra-thermal streams. If we limit ourselves by considering the electrons moving away from the Sun, the maximum expected effect is a redshift observable for positive slope angles $\Phi$. We see that this effect may become measurable for relatively moderate energies of electrons, and the diagnostics will be attempted in a forthcoming paper, using eclipse images.

Acknowledgements. The work is made in the frameworks of the French-Armenian "Jumelage" collaborative program, and we thank the French MAE and the Scientific Committee for providing resources to make this research.

\section{References}

Aharonian, F. A., \& Atoyan, A. M. 1981, Ap\&SS, 79, 321

Akhiezer, A. I., \& Berestetski, V. B. 1959, Quantum Electrodynamics (Fizmatgiz, M., in Russian)

Aschwanden, M. J., Benz, A. O., Dennis, B. R., \& Schwartz, R. A. 1995, ApJ, 455, 347

Berestetski, V. B., Lifshits, E. M., \& Pitaevski, L. P. 1989, Quantum Electrodynamics (Nauka, M., in Russian)

Billings, D. E. 1966, A Guide to the Solar Corona (Acad. Press, New York)

Buttighoffer, A. 1998, A\&A, 335, 295

Dzubenko, N. I., Ivanchuk, V. I., Nesmyanovich, A. T., \& Rubo, G. A. 1970, Astron Circ., 573 (in Russian)

Eddy, J. A. 1973, Sol. Phys., 30, 385

Eddy, J. A. 1979, A New Sun: The Solar Results from Skylab (NASA SP-402)

Fainberg, J., \& Stone, R. G. 1971, Sol. Phys., 17, 392

Harrison, R. 1986, A\&A, 162, 283

Hartz, T. R. 1969, Planet Space Sci., 17, 267

Haruthyunian, H. A., \& Nikoghossian, A. G. 1980, Doklady AN SSSR, 225, 86

Haruthyunian, H. A., \& Nikoghossian, A. G. 1981, in Invariance Principle and Its Applications (Yerevan), 431

Klein, K.-L., Aurass, H., Soru-Escaut, I., \& Kalman, B. 1997, A\&A, 322,1027

Kosovychev, A., \& Zharkova, V. 2001, ApJ, 550, L105

Koutchmy, S. 1969, Astrophys. Lett., 3, 215

Koutchmy, S. 1977, in Illustrated Glossary for Solar-terrestrial Physics, ed. A. Bruzek, \& C. J. Durrant (Reidel Publ. Co.)

Koutchmy, S. 1987, in Solar and Stellar Coronal Structures and Dynamics, Proc. of SPO Workshop, ed. R. Altrock

Lorrain, P., \& Koutchmy, S. 1996, Sol. Phys., 165, 115

Melendez, J. L., Sawant, H. S., Fernandes, F. C. R., \& Benz, A. O. 1999, Sol. Phys., 187, 77

Newkirk, G. 1967, ARA\&A, 5, 217

Nikoghossian, A. G., \& Koutchmy, S. 2001, Afz, 44, 643

Pick, M., \& van Den Oord, G. H. J. 1990, Sol. Phys., 130, 83

Rybicky, G. B., \& Lightman, A. P. 1979, in Radiative Processes in Astrophysics (Wiley, New York)

Thejappa, G., Goldstein, M. L., MacDowall, R. J., Papadopoulos, K., \& Stone, R. G. 1999, J. Geophys. Res., 104, 279

Vsessviatsky, S., \& Bougoslavskaya, E. 1944, MNRAS, 104, 140

Yokoyama, T., \& Shibata, K. 1996, PASJ, 48, 353 